PROCEEDINGS OF THE

AMERICAN MATHEMATICAL SOCIETY

Volume 139, Number 3, March 2011, Pages 943-956

S 0002-9939(2010)10532-4

Article electronically published on July 28, 2010

\title{
ILL-POSEDNESS RESULTS FOR THE (GENERALIZED) BENJAMIN-ONO-ZAKHAROV-KUZNETSOV EQUATION
}

\author{
AMIN ESFAHANI AND ADEMIR PASTOR
}

(Communicated by Hart F. Smith)

\begin{abstract}
Here we consider results concerning ill-posedness for the Cauchy problem associated with the Benjamin-Ono-Zakharov-Kuznetsov equation, namely,

(IVP)

$$
\left\{\begin{array}{l}
u_{t}-\mathcal{H} u_{x x}+u_{x y y}+u^{k} u_{x}=0, \quad(x, y) \in \mathbb{R}^{2}, \quad t \in \mathbb{R}^{+}, \\
u(x, y, 0)=\phi(x, y) .
\end{array}\right.
$$

For $k=1$, (IVP) is shown to be ill-posed in the class of anisotropic Sobolev spaces $H^{s_{1}, s_{2}}\left(\mathbb{R}^{2}\right), s_{1}, s_{2} \in \mathbb{R}$, while for $k \geq 2$ ill-posedness is shown to hold in $H^{s_{1}, s_{2}}\left(\mathbb{R}^{2}\right), 2 s_{1}+s_{2}<3 / 2-2 / k$. Furthermore, for $k=2,3$, and some particular values of $s_{1}, s_{2}$, a stronger result is also established.
\end{abstract}

\section{INTRODUCTION}

The propagation of two-dimensional dispersive weakly nonlinear waves is usually obtained by assuming nearly one-dimensional waves. As a result, there are several two-dimensional models which are generalizations of well-known one-dimensional nonlinear dispersive equations. The most known and studied ones are the Kadomtsev-Petviashvili (KP) and Zakharov-Kuznetsov equations, which are generalizations of the Korteweg-de Vries (KdV) equation.

The one-dimensional generalized Benjamin-Ono (BO) equation,

$$
u_{t}-\mathcal{H} u_{x x}+u^{k} u_{x}=0, \quad x \in \mathbb{R}, \quad t \in \mathbb{R}^{+},
$$

is a model for propagation of one-dimensional internal waves in an ideal deep stratified fluid (for $k=1$ ). In this paper we are interested in a model which is a natural two-dimensional extension of (1.1), namely, the generalized Benjamin-OnoZakharov-Kuznetsov (BO-ZK) equation,

$$
u_{t}-\mathcal{H} u_{x x}+\varepsilon u_{x y y}+u^{k} u_{x}=0, \quad(x, y) \in \mathbb{R}^{2}, \quad t \in \mathbb{R}^{+} .
$$

Here, $k>0$ is an integer number, the constant $\varepsilon$ measures the transverse effects and it is normalized to \pm 1 , and $\mathcal{H}$ is the Hilbert transform defined by

$$
\mathcal{H} u(x, y, t)=\text { p.v. } \frac{1}{\pi} \int_{\mathbb{R}} \frac{u(z, y, t)}{x-z} d z,
$$

Received by the editors January 15, 2010 and, in revised form, March 22, 2010.

2010 Mathematics Subject Classification. Primary 35Q51, 35Q53; Secondary 35Q35.

Key words and phrases. Nonlinear PDE, Cauchy problem, ill-posedness.

The first author was supported by FAPESP/SP-Brazil grant 2008/58892-6.

The second author was supported by CNPq-Brazil grant 152234/2007-1.

(C)2010 American Mathematical Society Reverts to public domain 28 years from publication 
where p.v. denotes the Cauchy principal value.

With $k=1$, equation (1.2) was introduced recently in [15, 17, and it has applications to electromigration in thin nanoconductors on a dielectric substrate.

Throughout the paper we consider $\varepsilon=1$, so that (1.2) reads as

$$
u_{t}-\mathcal{H} u_{x x}+u_{x y y}+u^{k} u_{x}=0 .
$$

Our main interest in the present paper lies in the study of the well-posedness (or ill-posedness) for the Cauchy problem associated with (1.3), so that we couple (1.3) with the Cauchy data

$$
u(x, y, 0)=\phi(x, y), \quad(x, y) \in \mathbb{R}^{2} .
$$

As usual, the well-posedness is taken to be in Kato's sense; that is, it includes existence, uniqueness, persistency property, and regularity (at least continuity) of the flow-map data-solution. Actually, our results will be negative ones in the sense that one cannot obtain high regularity of the map data-solution.

So far, not so much is known about equation (1.2), and only a few works are available in the literature. Indeed, concerning local well-posedness the best known result is the following.

Theorem 1.1. Let $s>2$. Then for any $\phi \in H^{s}\left(\mathbb{R}^{2}\right)$, there exist a positive $T=$ $T\left(\|\phi\|_{H^{s}}\right)$ and a unique solution $u \in C\left([0, T] ; H^{s}\left(\mathbb{R}^{2}\right)\right)$ of the Cauchy problem (1.2)(1.4). In addition, the flow-map $\phi \mapsto u(t)$ is continuous in the $H^{s}$-norm.

Theorem 1.1 will be not proved here, but it can be established just by using a parabolic regularization argument (see [19] for a similar result), so that it does not take into account the dispersive structure of the equation. It seems not to be easy to obtain a reasonable improvement of Theorem 1.1. We observe that recently Tao [28, Burq and Planchon [7, and Ionescu and Kenig [13] have obtained much stronger results for the Cauchy problem associated with the BO equation. However, it should be pointed out that their results are established by constructing appropriate gauge transformations. In the case of the BO-ZK equation, it is not clear how to get a suitable transformation, and we do not know if such an approach could be used to improve Theorem 1.1. On the other hand, we believe Theorem 1.1 can be improved by employing the technique introduced by Kenig [16] (see also [14] and [18]), which combines localized Strichartz estimates with some energy estimates. This will appear in a forthcoming paper.

In [8, 9], we studied the existence and stability of solitary-wave solutions of the form $u(x, y, t)=\varphi_{c}(x-c t, y), c>0$, in terms of the sign of $\varepsilon$ and the values of $k$ (even if $k$ is a rational number). To be more precise, for $\varepsilon=1$ and $0<k<4$, solitary waves do exist. By using the pioneer theory introduced by Cazenave and Lions, we have proved that such solitary waves are orbitally stable for $0<k<4 / 3$ (see [9]). It should be pointed out that Theorem 1.1 is not strong enough to consider global perturbations in the energy space $H^{1 / 2,1}\left(\mathbb{R}^{2}\right)$ (see the notation below). Thus, our stability result is in the sense that if a solution in $H^{s}\left(\mathbb{R}^{2}\right), s>2$, starts near the orbit generated by a solitary wave (in the $H^{1 / 2,1}\left(\mathbb{R}^{2}\right)$-norm), then as long as the solution exists, it remains near the orbit (see also [1] and 2] and the references therein). Moreover, by using the adapted method put forward by the KdV equation, we proved that the solitary waves are orbitally unstable for $4 / 3<k<4$ (see [8]).

We now turn our attention to describing our results. To motivate our interest in showing a local well-posedness, we first note that (1.3) has formally two conserved 
quantities, namely,

$$
\mathcal{F}(u)=\frac{1}{2} \int_{\mathbb{R}^{2}} u^{2} d x d y
$$

and

$$
\mathcal{E}(u)=\frac{1}{2} \int_{\mathbb{R}^{2}}\left(u_{y}^{2}+u \mathcal{H} u_{x}-\frac{2}{(k+1)(k+2)} u^{k+2}\right) d x d y .
$$

Thus, a local well-posedness result, in appropriate spaces, could lead to a global one.

Before trying to prove any result, we usually ask ourselves what would be the lowest isotropic (or anisotropic) Sobolev index where we can prove a local wellposedness result. To deal with this question, we perform a scaling argument by observing that if $u$ solves the equation (1.3) with initial data $\phi$, then

$$
u_{\lambda}(x, y, t)=\lambda^{2 / k} u\left(\lambda^{2} x, \lambda y, \lambda^{4} t\right)
$$

also solves (1.3) with initial data $u_{\lambda}(x, y, 0)=\lambda^{2 / k} \phi\left(\lambda^{2} x, \lambda y\right)$, for any $\lambda>0$. As a consequence, since

$$
\left\|u_{\lambda}(\cdot, \cdot, 0)\right\|_{\dot{H}^{s_{1}, s_{2}}}=\lambda^{2 s_{1}+s_{2}+2 / k-3 / 2}\|\phi\|_{\dot{H}^{s_{1}, s_{2}}},
$$

the scale-invariant Sobolev spaces for the BO-ZK equation are $\dot{H}^{s_{1}, s_{2}}\left(\mathbb{R}^{2}\right)$ with $2 s_{1}+s_{2}=3 / 2-2 / k$ (see the notation below). Thus, the natural spaces for studying the local well-posedness of equation (1.2) are the spaces $H^{s_{1}, s_{2}}\left(\mathbb{R}^{2}\right)$ with $2 s_{1}+s_{2} \geq 3 / 2-2 / k$.

We begin our results by considering the case $k=1$. Therefore, from the above scaling arguments, one expects to prove local well-posedness in the spaces $H^{s_{1}, s_{2}}\left(\mathbb{R}^{2}\right)$ with $2 s_{1}+s_{2} \geq-1 / 2$. However, by requiring $C^{2}$-regularity of the flowmap data-solution, we prove that one cannot obtain such results for any $s_{1}, s_{2} \in \mathbb{R}$. More precisely, we prove the following.

Theorem 1.2. Assume $k=1$. Let $s_{1}, s_{2} \in \mathbb{R}$ and $T>0$ be fixed numbers. Then, there does not exist a space $X_{T}$ continuously embedded in $C\left([0, T] ; H^{s_{1}, s_{2}}\left(\mathbb{R}^{2}\right)\right)$ such that there exists a constant $C>0$ with

$$
\|U(t) \phi\|_{X_{T}} \leq C\|\phi\|_{H^{s_{1}, s_{2}}}, \quad \phi \in H^{s_{1}, s_{2}}\left(\mathbb{R}^{2}\right),
$$

and

$$
\left\|\int_{0}^{t} U\left(t-t^{\prime}\right)\left[u\left(t^{\prime}\right) u_{x}\left(t^{\prime}\right)\right] d t^{\prime}\right\|_{X_{T}} \leq C\|u\|_{X_{T}}^{2}, \quad u \in X_{T},
$$

where $U(t)$ is the unitary group on $H^{s_{s}, s_{2}}\left(\mathbb{R}^{2}\right)$ defined, via its Fourier transform, by

$$
\widehat{U(t) \phi}(\xi, \eta)=\exp \left(i t\left(\xi|\xi|+\xi \eta^{2}\right)\right) \widehat{\phi}(\xi, \eta) .
$$

Corollary 1.3. Assume $k=1$. Let $s_{1}, s_{2} \in \mathbb{R}$ be fixed. Then there does not exist a $T>0$ such that the Cauchy problem (1.3) -(1.4) has a unique local solution defined on the interval $[0, T]$ and such that the flow-map data-solution

$$
S_{t}: \phi \longmapsto u(t), \quad t \in[0, T],
$$

is $C^{2}$-differentiable at the origin from $H^{s_{1}, s_{2}}\left(\mathbb{R}^{2}\right)$ to $H^{s_{1}, s_{2}}\left(\mathbb{R}^{2}\right)$. 
The main point to prove Theorem 1.2 is to explore the known results for the $\mathrm{BO}$ equation (1.1). Indeed, by using the technique introduced in [6] (see also [27]), it was proved in [23] that the $\mathrm{BO}$ equation is ill-posed in $H^{s}(\mathbb{R}), s \in \mathbb{R}$ (in the $C^{2}$-regularity sense). The main idea to prove such a result is to locate some special waves, in which, in some sense, the interaction between low and high frequencies behaves badly itself. Here, our insight comes from the physical viewpoint and, roughly speaking, it is the following. Since equation (1.3) is obtained under the assumption of nearly one-dimensional waves, we construct particular waves such that in the direction of propagation (the $x$-direction) they behave as the waves of the $\mathrm{BO}$ equation, whereas in the transverse direction (the $y$-direction), we localize them into small frequencies (see [19], 20] for similar ideas).

Remark 1.4. We note that the same proof of Theorem 1.2 (and Corollary 1.3) still holds when we replace the space $H^{s_{1}, s_{2}}\left(\mathbb{R}^{2}\right)$ with $H^{s}\left(\mathbb{R}^{2}\right), s \in \mathbb{R}$.

Next, we consider $k \geq 2$. In this case, our results are not so strong as in Theorem 1.2. However, for indices below the critical ones (in the scaling argument sense), we are able to show an ill-posedness result.

Theorem 1.5. Let $k \geq 2$ and $s_{1}, s_{2} \in \mathbb{R}$ such that $2 s_{1}+s_{2}<3 / 2-2 / k$. Then for any $T>0$, the flow-map $\phi \mapsto u$ (if it exists) is not of class $C^{k+1}$ from $H^{s_{1}, s_{2}}\left(\mathbb{R}^{2}\right)$ to $C\left([0, T] ; H^{s_{1}, s_{2}}\left(\mathbb{R}^{2}\right)\right)$ at the origin.

As in Theorem 1.2, the insight to show Theorem 1.5 is to explore the results for the generalized BO equation 21 (see also 3 and 22). Of course, some matters appear with the transverse direction, but, as in Theorem 1.2, we are able to handle them with suitable localizations.

Finally, we prove a result showing that for $k=2,3$ the flow-map data-solution cannot be uniformly continuous below the critical family of spaces for some values of $s_{1}, s_{2} \in \mathbb{R}$.

Theorem 1.6. Let $k=2,3$ and $s_{1}, s_{2} \geq 0$ such that $2 s_{1}+s_{2}=3 / 2-2 / k$. Then, the Cauchy problem (1.3)-(1.4) is ill-posed for data in $H^{s_{1}, s_{2}}\left(\mathbb{R}^{2}\right)$, in the sense that the flow-map data-solution, $\phi \mapsto u(t)$, is not uniformly continuous.

The method to prove Theorem 1.6 goes back to the techniques introduced in [4] and 5], which consists in constructing a sequence of converging data but such that the corresponding sequence of solutions does not converge. Usually, and this is our case, such data are given in terms of solitary-wave solutions. We note that the restriction on the values of $k$ lies in the fact that we do not know if solitary waves exist for $k \geq 4$ (see [8, 9]).

Remark 1.7. As is well known, our results imply that the Cauchy problem (1.3)(1.4) cannot be solved by an iterative method (in the respective spaces).

Notation. Throughout this paper we shall refer to equations (1.2) or (1.3) as the BO-ZK equation. For a function $f=f(x, y)$, the function $\widehat{f}=\widehat{f}(\xi, \eta)$ denotes its Fourier transform, defined as

$$
\widehat{f}(\xi, \eta)=\int_{\mathbb{R}^{2}} e^{-i(x \xi+y \eta)} f(x, y) d x d y .
$$

For any $s \in \mathbb{R}$, the space $H^{s}:=H^{s}\left(\mathbb{R}^{2}\right)$ denotes the usual isotropic Sobolev space. Let $s_{1}, s_{2} \in \mathbb{R}$. We define the anisotropic Sobolev spaces $H^{s_{1}, s_{2}}:=H^{s_{1}, s_{2}}\left(\mathbb{R}^{2}\right)$ to 
be the set of all tempered distributions $f$ such that

$$
\|f\|_{H^{s_{1}, s_{2}}}^{2}=\int_{\mathbb{R}^{2}}\left(1+\xi^{2}\right)^{s_{1}}\left(1+\eta^{2}\right)^{s_{2}}|\widehat{f}(\xi, \eta)|^{2} d \xi d \eta<\infty .
$$

The homogeneous anisotropic Sobolev space $\dot{H}^{s_{1}, s_{2}}:=\dot{H}^{s_{1}, s_{2}}\left(\mathbb{R}^{2}\right)$ is defined to be the set of all tempered distributions $f$ such that

$$
\|f\|_{\dot{H}^{s_{1}, s_{2}}}^{2}=\int_{\mathbb{R}^{2}}|\xi|^{2 s_{1}}|\eta|^{2 s_{2}}|\widehat{f}(\xi, \eta)|^{2} d \xi d \eta<\infty .
$$

\section{Proofs of Theorem 1.2 and Corollary 1.3}

In this section, we prove Theorem 1.2 and Corollary 1.3 . As we already mentioned, we follow closely the arguments in [23].

Let us first consider the linear problem

$$
\left\{\begin{array}{l}
u_{t}-\mathcal{H} u_{x x}+u_{x y y}=0, \quad(x, y) \in \mathbb{R}^{2}, \quad t>0 \\
u(x, y, 0)=\phi(x, y)
\end{array}\right.
$$

It is easily seen that the solution of (2.1) is given by

$$
u(t)=U(t) \phi(x, y)=\int_{\mathbb{R}^{2}} e^{i\left(t\left(\xi|\xi|+\xi \eta^{2}\right)+x \xi+y \eta\right)} \widehat{\phi}(\xi, \eta) d \xi d \eta
$$

Proof of Theorem 1.2. The proof is argued by contradiction. Indeed, assume that such a space does exist and that (1.5) and (1.6) are fulfilled. By taking $u(t)=U(t) \phi$ in (1.6), we obtain

$$
\left\|\int_{0}^{t} U\left(t-t^{\prime}\right)\left[\left(U\left(t^{\prime}\right) \phi\right)\left(U\left(t^{\prime}\right) \phi_{x}\right)\right] d t^{\prime}\right\|_{X_{T}} \leq C\|U(t) \phi\|_{X_{T}}^{2} .
$$

Since $X_{T}$ is continuously embedded in $C\left([0, T] ; H^{s_{1}, s_{2}}\left(\mathbb{R}^{2}\right)\right)$, (1.5) yields

$$
\left\|\int_{0}^{t} U\left(t-t^{\prime}\right)\left[\left(U\left(t^{\prime}\right) \phi\right)\left(U\left(t^{\prime}\right) \phi_{x}\right)\right] d t^{\prime}\right\|_{H^{s_{1}, s_{2}}} \leq C\|\phi\|_{H^{s_{1}, s_{2}}}^{2} .
$$

The idea now is to show that (2.3) fails by constructing a particular $\phi$.

For $0<\alpha \ll 1$ and $N \gg 1$, we consider the (disjoint) rectangles

$$
Q_{1}=[\alpha / 2, \alpha] \times[\sqrt{\alpha} / 2, \sqrt{\alpha}], \quad Q_{2}=[N, N+\alpha] \times[\sqrt{\alpha} / 2, \sqrt{\alpha}],
$$

and define $\phi$, via its Fourier transform, by

$$
\widehat{\phi}(\xi, \eta):=\alpha^{-\frac{3}{4}} \chi_{Q_{1}}+\alpha^{-\frac{3}{4}} N^{-s_{1}} \chi_{Q_{2}},
$$

where $\chi_{A}$ denotes the characteristic function of the set $A$.

Lemma 2.1. Let $\phi$ be as in (2.4) and $s_{1}, s_{2} \in \mathbb{R}$. Then, there exists a constant $K>0$, independent of $N$ and $\alpha$, such that

$$
\|\phi\|_{H^{s_{1}, s_{2}}} \leq K
$$

Proof. The proof is a straightforward calculation.

The following lemma is useful. 
Lemma 2.2. Let

$$
p(\xi, \eta)=\xi|\xi|+\xi \eta^{2}
$$

The following identity holds:

$$
\begin{aligned}
& \int_{0}^{t} U\left(t-t^{\prime}\right)\left[\left(U\left(t^{\prime}\right) \phi\right)\left(U\left(t^{\prime}\right) \phi_{x}\right)\right] d t^{\prime} \\
& \quad=\int_{\mathbb{R}^{4}} e^{i(x \xi+y \eta+t p(\xi, \eta))} \hat{\phi}\left(\xi_{1}, \eta_{1}\right) \widehat{\phi}\left(\xi-\xi_{1}, \eta-\eta_{1}\right) \frac{e^{i t \Lambda\left(\xi, \xi_{1}, \eta, \eta_{1}\right)}-1}{\Lambda\left(\xi, \xi_{1}, \eta, \eta_{1}\right)} d \xi d \xi_{1} d \eta d \eta_{1},
\end{aligned}
$$

where

$$
\Lambda\left(\xi, \xi_{1}, \eta, \eta_{1}\right)=p\left(\xi_{1}, \eta_{1}\right)+p\left(\xi-\xi_{1}, \eta-\eta_{1}\right)-p(\xi, \eta) .
$$

Proof. The proof is carried out in a similar way to that in [23, Lemma 1] and [24, Lemma 4].

In view of Lemma 2.2 we write

$$
\int_{0}^{t} U\left(t-t^{\prime}\right)\left[\left(U\left(t^{\prime}\right) \phi\right)\left(U\left(t^{\prime}\right) \phi_{x}\right)\right] d t^{\prime}=f_{1}(x, y, t)+f_{2}(x, y, t)+f_{3}(x, y, t)
$$

where

$$
\begin{gathered}
f_{1}(x, y, t)=\frac{c}{\alpha^{3 / 2}} \int_{Q_{11}} e^{i(x \xi+y \eta+t p(\xi, \eta))} \xi \frac{e^{i t \Lambda\left(\xi, \xi_{1}, \eta, \eta_{1}\right)}-1}{\Lambda\left(\xi, \xi_{1}, \eta, \eta_{1}\right)} d \xi d \xi_{1} d \eta d \eta_{1}, \\
f_{2}(x, y, t)=\frac{c}{\alpha^{3 / 2} N^{2 s_{1}}} \int_{Q_{22}} e^{i(x \xi+y \eta+t p(\xi, \eta))} \xi \frac{e^{i t \Lambda\left(\xi, \xi_{1}, \eta, \eta_{1}\right)}-1}{\Lambda\left(\xi, \xi_{1}, \eta, \eta_{1}\right)} d \xi d \xi_{1} d \eta d \eta_{1},
\end{gathered}
$$

and

$$
f_{3}(x, y, t)=\frac{c}{\alpha^{3 / 2} N^{s_{1}}} \int_{Q_{12} \cup Q_{21}} e^{i(x \xi+y \eta+t p(\xi, \eta))} \xi \frac{e^{i t \Lambda\left(\xi, \xi_{1}, \eta, \eta_{1}\right)}-1}{\Lambda\left(\xi, \xi_{1}, \eta, \eta_{1}\right)} d \xi d \xi_{1} d \eta d \eta_{1} .
$$

Here, we have defined

$$
\begin{aligned}
& Q_{11}:=\left\{\left(\xi, \xi_{1}, \eta, \eta_{1}\right) \in \mathbb{R}^{4} ;\left(\xi_{1}, \eta_{1}\right) \in Q_{1},\left(\xi-\xi_{1}, \eta-\eta_{1}\right) \in Q_{1}\right\}, \\
& Q_{22}:=\left\{\left(\xi, \xi_{1}, \eta, \eta_{1}\right) \in \mathbb{R}^{4} ;\left(\xi_{1}, \eta_{1}\right) \in Q_{2},\left(\xi-\xi_{1}, \eta-\eta_{1}\right) \in Q_{2}\right\}, \\
& Q_{12}:=\left\{\left(\xi, \xi_{1}, \eta, \eta_{1}\right) \in \mathbb{R}^{4} ;\left(\xi_{1}, \eta_{1}\right) \in Q_{1},\left(\xi-\xi_{1}, \eta-\eta_{1}\right) \in Q_{2}\right\},
\end{aligned}
$$

and

$$
Q_{21}:=\left\{\left(\xi, \xi_{1}, \eta, \eta_{1}\right) \in \mathbb{R}^{4} ;\left(\xi_{1}, \eta_{1}\right) \in Q_{2},\left(\xi-\xi_{1}, \eta-\eta_{1}\right) \in Q_{1}\right\} .
$$

Note that taking the Fourier transform (in the variables $x, y$ ), we obtain

$$
\begin{gathered}
\widehat{f}_{1}(\xi, \eta, t)=\frac{c}{\alpha^{3 / 2}} \xi e^{i t p(\xi, \eta)} \int_{\left(\xi_{1}, \eta_{1}\right) \in Q_{11}} \frac{e^{i t \Lambda\left(\xi, \xi_{1}, \eta, \eta_{1}\right)}-1}{\Lambda\left(\xi, \xi_{1}, \eta, \eta_{1}\right)} d \xi_{1} d \eta_{1}, \\
\widehat{f}_{2}(\xi, \eta, t)=\frac{c}{\alpha^{3 / 2} N^{2 s_{1}}} \xi e^{i t p(\xi, \eta)} \int_{\left(\xi_{1}, \eta_{1}\right) \in Q_{22}} \frac{e^{i t \Lambda\left(\xi, \xi_{1}, \eta, \eta_{1}\right)}-1}{\Lambda\left(\xi, \xi_{1}, \eta, \eta_{1}\right)} d \xi_{1} d \eta_{1},
\end{gathered}
$$

and

$$
\widehat{f_{3}}(\xi, \eta, t)=\frac{c}{\alpha^{3 / 2} N^{s_{1}}} \xi e^{i t p(\xi, \eta)} \int_{\left(\xi_{1}, \eta_{1}\right) \in Q_{12} \cup Q_{21}} \frac{e^{i t \Lambda\left(\xi, \xi_{1}, \eta, \eta_{1}\right)}-1}{\Lambda\left(\xi, \xi_{1}, \eta, \eta_{1}\right)} d \xi_{1} d \eta_{1} .
$$

Next, we analyze the supports of $\widehat{f}_{j}, j=1,2,3$. Actually, a simple analysis shows us that

(1) $\operatorname{supp}\left(\widehat{f}_{1}\right) \subset[\alpha, 2 \alpha] \times[\sqrt{\alpha}, 2 \sqrt{\alpha}]$, 
(2) $\operatorname{supp}\left(\widehat{f}_{2}\right) \subset[2 N, 2 N+2 \alpha] \times[\sqrt{\alpha}, 2 \sqrt{\alpha}]$,

(3) $\operatorname{supp}\left(\widehat{f}_{3}\right) \subset[N, N+2 \alpha] \times[\sqrt{\alpha}, 2 \sqrt{\alpha}]$.

Since the supports of $\widehat{f}_{j}, j=1,2,3$ are disjoint, from (2.5), we deduce

$$
\left\|\int_{0}^{t} U\left(t-t^{\prime}\right)\left[\left(U\left(t^{\prime}\right) \phi\right)\left(U\left(t^{\prime}\right) \phi_{x}\right)\right] d t^{\prime}\right\|_{H^{s_{1}, s_{2}}} \geq\left\|f_{3}(\cdot, \cdot, t)\right\|_{H^{s_{1}, s_{2}}} .
$$

The aim now is to get some "good" lower bound for $\left\|f_{3}(\cdot, \cdot, t)\right\|_{H^{s}}$. We first note that if $\left(\xi, \xi_{1}, \eta, \eta_{1}\right) \in Q_{12} \cup Q_{21}$, then

$$
\Lambda\left(\xi, \xi_{1}, \eta, \eta_{1}\right)=-\left[\left(\xi-\xi_{1}\right)\left(2 \xi_{1}+\eta \eta_{1}\right)+\left(\eta-\eta_{1}\right)\left(\xi \eta_{1}+\xi_{1} \eta\right)\right] .
$$

Therefore, for $\left(\xi, \xi_{1}, \eta, \eta_{1}\right) \in Q_{12} \cup Q_{21}$, we have

$$
c \alpha N \leq\left|\Lambda\left(\xi, \xi_{1}, \eta, \eta_{1}\right)\right| \leq C \alpha N,
$$

where $c$ and $C$ are constants independent of $\alpha$ and $N$. Thus, we choose $\alpha$ and $N$ such that

$$
\alpha N=N^{-\epsilon}, \quad 0<\epsilon \ll 1 .
$$

Now, for $\left(\xi, \xi_{1}, \eta, \eta_{1}\right) \in Q_{12} \cup Q_{21}$, from (2.7), we obtain

$$
\left|\frac{e^{i t \Lambda\left(\xi, \xi_{1}, \eta, \eta_{1}\right)}-1}{\Lambda\left(\xi, \xi_{1}, \eta, \eta_{1}\right)}\right|=|t|+O\left(N^{-\epsilon}\right) .
$$

Hence, using the integral mean value theorem, we get the bound

$$
\left\|f_{3}(\cdot, \cdot, t)\right\|_{H^{s, s_{2}}} \geq C \frac{N N^{s_{1}} \alpha^{3 / 2} \alpha^{3 / 4}}{N^{s_{1}} \alpha^{3 / 2}}=C \alpha^{3 / 4} N=C N^{(1-3 \epsilon) / 4} .
$$

Finally, gathering together Lemma 2.1 and inequalities (2.3), (2.6), and (2.8), we get

$$
K^{2} \geq\|\phi\|_{H^{s_{1}, s_{2}}}^{2} \geq\left\|f_{3}(\cdot, \cdot, t)\right\|_{H^{s_{1}, s_{2}}} \geq C N^{(1-3 \epsilon) / 4},
$$

which is a contradiction for $N \gg 1$. The proof of Theorem 1.2 is now completed.

Proof of Corollary 1.3. This is well understood by now. If the flow-map datasolution was $C^{2}$-differentiable at the origin from $H^{s_{1}, s_{2}}\left(\mathbb{R}^{2}\right)$ to $H^{s_{1}, s_{2}}\left(\mathbb{R}^{2}\right)$, we should obtain

$$
\left\|\int_{0}^{t} U\left(t-t^{\prime}\right)\left[\left(U\left(t^{\prime}\right) \phi\right)\left(U\left(t^{\prime}\right) \phi_{x}\right)\right] d t^{\prime}\right\|_{H^{s_{1}, s_{2}}} \leq C\|\phi\|_{H^{s_{1}, s_{2}}}^{2} .
$$

But as we showed in Theorem 1.2, the above inequality fails. This proves Corollary 1.3 .

2.1. Generalizations to a class of dispersive equations. In this subsection, we consider a general class of dispersive equations, for which the same conclusions of Theorem 1.2 and Corollary 1.3 hold. Indeed, we consider the Cauchy problem

$$
\left\{\begin{array}{l}
u_{t}-\mathcal{L} u+u u_{x}=0, \\
u(x, y, 0)=\phi(x, y)
\end{array} \quad(x, y) \in \mathbb{R}^{2}, \quad t>0\right.
$$

where $\mathcal{L}$ is a Fourier multiplier given by

$$
\widehat{\mathcal{L f}}(\xi, \eta)=i p(\xi, \eta) \widehat{f}(\xi, \eta) .
$$

The following assumptions will be needed in the remainder of this section.

(H1) The symbol $p$ is a continuous real-valued function on $\mathbb{R}^{2}$ and differentiable on $\mathbb{R}^{+} \times \mathbb{R}^{+}$. 
(H2) $p(0,0)=0$, and if $\nabla p=\left(p_{1}, p_{2}\right)$, then

$$
\left|p_{j}(\xi, \eta)\right| \lesssim|\eta|^{a_{j}}\left(|\xi|^{\gamma_{j}}+|\eta|^{b_{j}}\right), \quad(\xi, \eta) \in \mathbb{R}^{+} \times \mathbb{R}^{+},
$$

with $0 \leq \gamma_{j}<4 / 3, b_{j} \geq 0, j=1,2, a_{1} \geq 0$, and $a_{2} \geq 1$.

Lemma 2.3. Let assumptions (H1)-(H2) hold. Then for $\left(\xi, \xi_{1}, \eta, \eta_{1}\right) \in Q_{12} \cup Q_{21}$, we have

$$
\left|\Lambda\left(\xi, \xi_{1}, \eta, \eta_{1}\right)\right| \leq C \alpha N^{\gamma}, \quad \alpha \ll 1, \quad N \gg 1,
$$

where $\gamma=\max \left\{\gamma_{1}, \gamma_{2}\right\}, Q_{12}, Q_{21}$ are as in the previous section, and

$$
\Lambda\left(\xi, \xi_{1}, \eta, \eta_{1}\right)=p\left(\xi_{1}, \eta_{1}\right)+p\left(\xi-\eta, \xi_{1}-\eta_{1}\right)-p(\xi, \eta) .
$$

Proof. We first note that from the mean value theorem,

$$
|p(\xi, \eta)| \lesssim|\eta|^{a_{1}}|\xi|\left(|\xi|^{\gamma_{1}}+|\eta|^{b_{1}}\right)+|\eta|^{a_{2}+1}\left(|\xi|^{\gamma_{2}}+|\eta|^{b_{2}}\right)
$$

for $(\xi, \eta) \in \mathbb{R}^{+} \times \mathbb{R}^{+}$. Now (as in [12]), we denote

$$
\left|\xi_{\min }\right|=\min \left\{|\xi|,\left|\xi_{1}\right|,\left|\xi-\xi_{1}\right|\right\}, \quad\left|\xi_{\max }\right|=\max \left\{|\xi|,\left|\xi_{1}\right|,\left|\xi-\xi_{1}\right|\right\} .
$$

Suppose first that $\left|\xi_{\min }\right|=\xi_{1}$. Then, for some $\theta \in[0,1]$, we have

$$
\begin{aligned}
& \left|p(\xi, \eta)-p\left(\xi-\xi_{1}, \eta-\eta_{1}\right)\right| \\
& \lesssim\left|\eta-\theta \eta_{1}\right|^{a_{1}}\left|\xi_{1}\right|\left(\left|\xi-\theta \xi_{1}\right|^{\gamma_{1}}+\left|\eta-\theta \eta_{1}\right|^{b_{1}}\right) \\
& +\left|\eta-\theta \eta_{1}\right|^{a_{2}}\left|\eta_{1}\right|\left(\left|\xi-\theta \xi_{1}\right|^{\gamma_{2}}+\left|\eta-\theta \eta_{1}\right|^{b_{2}}\right) \\
& \lesssim\left|\eta-\theta \eta_{1}\right|^{a_{1}}\left|\xi_{\min }\right|\left(\left|\xi_{\max }\right|^{\gamma_{1}}+\left|\eta-\theta \eta_{1}\right|^{b_{1}}\right)+\left|\eta-\theta \eta_{1}\right|^{a_{2}}\left|\eta_{1}\right|\left(\left|\xi_{\max }\right|^{\gamma_{2}}+\left|\eta-\theta \eta_{1}\right|^{b_{2}}\right),
\end{aligned}
$$

where we have used the fact that

$$
\max _{\theta \in[0,1]}\left|\xi-\theta \xi_{1}\right|=\left|\xi_{\max }\right|
$$

Moreover, from (2.10),

$$
\left|p\left(\xi_{1}, \eta_{1}\right)\right| \lesssim\left|\eta_{1}\right|^{a_{1}}\left|\xi_{\min }\right|\left(\left|\xi_{\max }\right|^{\gamma_{1}}+\left|\eta_{1}\right|^{b_{1}}\right)+\left|\eta_{1}\right|^{a_{2}+1}\left(\left|\xi_{\max }\right|^{\gamma_{2}}+\left|\eta_{1}\right|^{b_{2}}\right) .
$$

Therefore, the structure of $Q_{12}$ and $Q_{21}$ imply that for $\left(\xi, \xi_{1}, \eta, \eta_{1}\right) \in Q_{12} \cup Q_{21}$,

$$
\left|\Lambda\left(\xi, \xi_{1}, \eta, \eta_{1}\right)\right| \leq\left|p\left(\xi_{1}, \eta_{1}\right)\right|+\left|p\left(\xi-\eta, \xi_{1}-\eta_{1}\right)-p(\xi, \eta)\right| \lesssim \alpha N^{\gamma} .
$$

If $\left|\xi_{\min }\right|=\left|\xi-\xi_{1}\right|$, a similar analysis can be performed. Note that in $Q_{12} \cup Q_{21}$ we always have $|\xi| \geq \max \left\{\left|\xi_{1}\right|, \mid \xi-\xi_{1}\right\}$. This completes the proof of the lemma.

Theorem 2.4. Let assumptions (H1)-(H2) hold. Then the conclusions of Theorem 1.2 and Corollary 1.3 are valid for the Cauchy problem (2.9).

Sketch of the proof. In this case one chooses $\alpha$ and $N$ such that $\alpha N^{\gamma}=N^{-\epsilon}, 0<$ $\epsilon \ll 1$. Now, similarly to the proof of Theorem 1.2 , we obtain

$$
K^{2} \geq\|\phi\|_{H^{s_{1}, s_{2}}}^{2} \geq C \alpha^{3 / 4} N=C N^{(4-3 \gamma-3 \epsilon) / 4},
$$

which is a contradiction under our assumptions. 
2.1.1. Examples. It is easy to see that the BO-ZK equation (1.2) fits in the hypotheses of Theorem 2.4. Here, we give another example where assumptions (H1)-(H2) are verified. The Shrira equation (see [26])

$$
u_{t}+\mathcal{H} \Delta u+u u_{x}=0
$$

where $\Delta=\partial_{x}^{2}+\partial_{y}^{2}$ denotes the Laplacian operator, is a model for the description of essentially two-dimensional weakly nonlinear long-wave perturbations on the background of a boundary-layer type plane-parallel shear flow without inflection points (see [25]). It also describes the amplitude of the perturbation of the horizontal velocity component of a sheared flow of electrons (see [11]). For existence and stability of solitary waves, see [10].

In this case, we have

$$
p(\xi, \eta)=\operatorname{sign}(\xi)\left(\xi^{2}+\eta^{2}\right) .
$$

A trivial verification shows that assumptions (H1)-(H2) hold.

\section{Proof of Theorem 1.5}

In this section, we show Theorem 1.5. As previously commented, we use the adapted method put forward in 21] for the generalized BO equation.

Let us consider the flow-map $\phi \mapsto u(t ; \phi)$, and define $u_{k+1}$ by

$$
u_{k+1}=\left.\frac{\partial^{k+1} u}{\partial^{k+1} \phi}\right|_{\phi=0}\left(h_{N}, \ldots, h_{N}\right),
$$

where the sequence $\left\{h_{N}\right\}$ will be constructed below. Then, since $u(\cdot ; 0)=0$, by straightforward calculations, we see that

$$
u_{k+1}=(k+1) ! \int_{0}^{t} U\left(t-t^{\prime}\right) \partial_{x}\left(\left(U\left(t^{\prime}\right) h_{N}\right)^{k+1}\right) d t^{\prime} .
$$

Thus, if $\phi \mapsto u(\cdot ; \phi)$ is of class $C^{k+1}$ at the origin, we see that necessarily

$$
\sup _{t \in[0, T]}\left\|u_{k+1}(t)\right\|_{H^{s_{1}, s_{2}}} \leq C\left\|h_{N}\right\|_{H^{s_{1}, s_{2}}}^{k+1} .
$$

In the sequel, we show that (3.1) fails for a suitable sequence of functions $\left\{h_{N}\right\}_{N}$. Let $A$ and $B$ be positive real numbers (which will be chosen later) such that $A<B$ and $A>k B /(k+2)$. Consider the real-valued function $h_{N}$ defined, via its Fourier transform, by

$$
\widehat{h}_{N}(\xi, \eta)=N^{-\left(4 s_{1}+2 s_{2}+3\right) / 4}\left(\psi_{1+}+\psi_{1-}+\psi_{2+}+\psi_{2-}\right)\left(\frac{\xi}{N}, \frac{\eta}{\sqrt{N}}\right),
$$

where $\psi_{1+}$ is a smooth nonnegative function supported in the set

$$
\left\{(\xi, \eta) \in \mathbb{R}^{2} \mid 0<A \leq \xi, \eta \leq B\right\},
$$

$\psi_{1+} \equiv 1$ on the square

$$
\left[A+\frac{1}{4}(B-A), B-\frac{1}{4}(B-A)\right] \times\left[A+\frac{1}{4}(B-A), B-\frac{1}{4}(B-A)\right],
$$

and

$$
\psi_{1+}(\xi, \eta)=\psi_{1-}(-\xi, \eta)=\psi_{2+}(\xi,-\eta)=\psi_{2-}(-\xi,-\eta) .
$$

Note that by definition,

$$
\left\|h_{N}\right\|_{H^{s_{1}, s_{2}}} \simeq 1
$$


On the other hand, we see that the Fourier transform of $u_{k+1}$ can be computed as follows:

$$
\begin{aligned}
\widehat{u}_{k+1}( & \left.t, \xi_{0}, \eta_{0}\right) \chi_{[k A N, k B N] \times[k A \sqrt{N}, k B \sqrt{N}]} \\
= & N^{-\left(4 s_{1}+2 s_{2}+3\right)(k+1) / 4} e^{i t p\left(\xi_{0}, \eta_{0}\right)} \xi_{0} \int_{0}^{t} e^{-i t^{\prime} p(\xi, \eta)} \mathcal{F}_{\xi, \eta}\left(\left(U\left(t^{\prime}\right) \psi_{1+}\right)^{k+1}\right)\left(\xi_{0}, \eta_{0}\right) d t^{\prime} \\
= & N^{-\left(4 s_{1}+2 s_{2}+3\right)(k+1) / 4} e^{i t p\left(\xi_{0}, \eta_{0}\right)} i \xi_{0} \int_{\mathbb{R}^{2 k}} \frac{e^{-i t F\left(\xi_{0}, \eta_{0}, \ldots, \xi_{k}, \eta_{k}\right)}-1}{F\left(\xi_{0}, \eta_{0}, \ldots, \xi_{k}, \eta_{k}\right)} \\
& \times \psi_{1+}\left(\frac{\xi_{0}-\xi_{1}}{N}, \frac{\eta_{0}-\eta_{1}}{\sqrt{N}}\right) \cdots \psi_{1+}\left(\frac{\xi_{k-1}-\xi_{k}}{N}, \frac{\eta_{k-1}-\eta_{k}}{\sqrt{N}}\right) \\
& \times \psi_{1+}\left(\frac{\xi_{k}}{N}, \frac{\eta_{k}}{\sqrt{N}}\right) d \xi_{1} d \eta_{1} \cdots d \xi_{k} d \eta_{k},
\end{aligned}
$$

where $p\left(\xi_{0}, \eta_{0}\right)=\xi_{0}\left|\xi_{0}\right|+\xi_{0} \eta_{0}^{2}$ and

$$
\begin{aligned}
F\left(\xi_{0}, \eta_{0}, \ldots, \xi_{k}, \eta_{k}\right)= & \sum_{j=1}^{k} \xi_{j}\left[2\left(\xi_{j-1}-\xi_{j}\right)+\left(\eta_{j-1}-\eta_{j}\right)^{2}\right] \\
& +\sum_{j=1}^{k}\left(\xi_{j-1}-\xi_{j}\right) \eta_{j}^{2}+2 \xi_{j-1} \eta_{j}\left(\eta_{j-1}-\eta_{j}\right) .
\end{aligned}
$$

Note that in the subset of $\mathbb{R}^{2 k}$, defined by

$$
A \leq \frac{\xi_{0}-\xi_{1}}{N} \leq B, \ldots, A \leq \frac{\xi_{k-1}-\xi_{k}}{N} \leq B, \quad A \leq \frac{\xi_{k}}{N} \leq B
$$

and

$$
A \leq \frac{\eta_{0}-\eta_{1}}{\sqrt{N}} \leq B, \ldots, A \leq \frac{\eta_{k-1}-\eta_{k}}{\sqrt{N}} \leq B, \quad A \leq \frac{\eta_{k}}{\sqrt{N}} \leq B,
$$

we have that

$$
\frac{k(k+1)}{2}\left(A^{2}+A^{3}\right) N^{2} \leq F\left(\xi_{0}, \eta_{0}, \ldots, \xi_{k}, \eta_{k}\right) \leq \frac{k(k+1)}{2}\left(B^{2}+B^{3}\right) N^{2} .
$$

Thus, it follows that for

$$
t_{N}=\frac{2}{A^{2} k(k+1)} N^{-2} \simeq N^{-2}
$$

and $A$ close enough to $B$,

$$
\begin{aligned}
& \left|\widehat{u}_{k+1}\left(t_{N}, \xi_{0}, \eta_{0}\right)\right| \chi_{[k A N, k B N] \times\left[k A \sqrt{N}, k B \sqrt{N]} \geq N^{-\left(4 s_{1}+2 s_{2}+3\right)(k+1) / 4} N^{-2}\right.} \\
& \quad \times \int_{\mathbb{R}^{2 k}}\left|\xi_{0}\right| \psi_{1+}\left(\frac{\xi_{0}-\xi_{1}}{N}, \frac{\eta_{0}-\eta_{1}}{\sqrt{N}}\right) \cdots \psi_{1+}\left(\frac{\xi_{k-1}-\xi_{k}}{N}, \frac{\eta_{k-1}-\eta_{k}}{\sqrt{N}}\right) \\
& \quad \times \psi_{1+}\left(\frac{\xi_{k}}{N}, \frac{\eta_{k}}{\sqrt{N}}\right) d \xi_{1} d \eta_{1} \cdots d \xi_{k} d \eta_{k} .
\end{aligned}
$$

By using that for any $a, b, c, d \in \mathbb{R}$,

$$
\widehat{\chi}_{[a, b] \times[c, d]}(\xi, \eta)=4 \frac{\sin ((b-a) \xi / 2) \sin ((d-c) \eta / 2)}{\xi \eta} e^{-i((a+b) \xi+(c+d) \eta) / 2},
$$


we obtain that, for any $\varrho>0$,

$$
\begin{aligned}
& \chi_{\left[\omega_{1} N, \omega_{2} N\right] \times\left[\omega_{1} \sqrt{N}, \omega_{2} \sqrt{N}\right]} * \cdots * \chi_{\left[\omega_{1} N, \omega_{2} N\right] \times\left[\omega_{1} \sqrt{N}, \omega_{2} \sqrt{N}\right]}(N(\omega+\varrho), \sqrt{N}(\omega+\varrho)) \\
& =2^{2 k} N^{3(k-1) / 2} \int_{\mathbb{R}^{2}} \cos (\varrho \xi) \cos (\varrho \eta)\left(\frac{\sin (\tau \xi) \sin (\tau \eta)}{\xi \eta}\right)^{k} d \xi d \eta
\end{aligned}
$$

where

$$
\omega_{1}=A+\tau, \quad \omega_{2}=B-\tau, \quad \tau=\frac{1}{4}(B-A), \quad \text { and } \quad \omega=k\left(\omega_{1}+\omega_{2}\right) / 2 .
$$

As in 22], this proves that there exists $\varrho>0$, small enough, such that

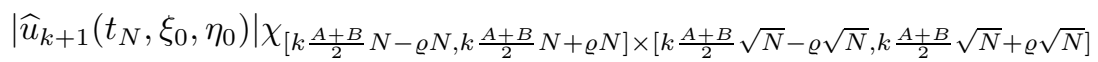

$$
\begin{aligned}
& \geq N^{-\left(4 s_{1}+2 s_{2}+3\right)(k+1) / 4} N^{-2}\left|\xi_{0}\right| N^{3 k / 2} \\
& \times \chi_{\left[k \frac{A+B}{2} N-\varrho N, k \frac{A+B}{2} N+\varrho N\right] \times\left[k \frac{A+B}{2} \sqrt{N}-\varrho \sqrt{N}, k \frac{A+B}{2} \sqrt{N}+\varrho \sqrt{N}\right]},
\end{aligned}
$$

which yields

$$
\left\|u_{k+1}\left(t_{N}\right)\right\|_{H^{s_{1}, s_{2}}}^{2} \geq C N^{-\left(4 s_{1}+2 s_{2}+3\right)(k+1) / 2} N^{3 k-4} N^{2 s_{1}+3} N^{s_{2}+\frac{1}{2}} .
$$

Hence, for $T>0$ fixed,

$$
\lim _{N \rightarrow+\infty} \sup _{t \in[0, T]}\left\|u_{k+1}(t)\right\|_{H^{s_{1}, s_{2}}} \rightarrow+\infty,
$$

as soon as $2 s_{1}+s_{2}<3 / 2-2 / k$. Thus, estimate (3.1) fails, and the proof of Theorem 1.5 is completed.

\section{Proof of Theorem 1.6}

In this section, attention is given to the proof of Theorem 1.6. First, let us recall some facts about solitary-wave solutions for (1.3). In order to describe our results, the space $Z$ shall denote the closure of $C_{0}^{\infty}\left(\mathbb{R}^{2}\right)$ with respect to the norm

$$
\|\varphi\|_{\mathcal{Z}}^{2}=\|\varphi\|_{L^{2}\left(\mathbb{R}^{2}\right)}^{2}+\left\|\varphi_{y}\right\|_{L^{2}\left(\mathbb{R}^{2}\right)}^{2}+\left\|\partial_{x}^{1 / 2} \varphi\right\|_{L^{2}\left(\mathbb{R}^{2}\right)}^{2},
$$

where $\partial_{x}^{1 / 2}$ denotes the fractional derivative of order $1 / 2$ with respect to $x$, defined, via its Fourier transform, by $\widehat{\partial_{x}^{1 / 2} \varphi}(\xi, \eta)=|\xi|^{1 / 2} \widehat{\varphi}(\xi, \eta)$.

Solitary waves are solutions of (1.3) of the form $u(x, y, t)=\varphi_{c}(x-c t, y)$, where $\varphi_{c} \in Z$ and $c \neq 0$ is the wave speed. Thus, substituting this form of $u$ in (1.3) and integrating once, we see that $\varphi:=\varphi_{c}$ must satisfy

$$
-c \varphi+\frac{1}{k+1} \varphi^{k+1}-\mathcal{H} \varphi_{x}+\varphi_{y y}=0 .
$$

The next lemma is sufficient for our purpose. For the proof we refer the reader to [9, where additional properties of the solitary waves are given.

Lemma 4.1. Assume $1 \leq k \leq 3$ and $c>0$. Then equation (4.2) admits a nontrivial solution $\varphi_{c} \in 2$. Moreover, $\varphi_{c} \in H^{\infty}\left(\mathbb{R}^{2}\right)$, and it decays algebraically in the $x$ direction and exponentially in the $y$-direction. 
Proof of Theorem 1.6. Assume $k=2$ or $k=3$. Let $s_{1}, s_{2} \geq 0$ be such that $2 s_{1}+$ $s_{2}=3 / 2-2 / k$. It is easy to see that

$$
\varphi_{c}(x, y)=c^{1 / k} \varphi_{1}(c x, \sqrt{c} y), \quad c>0 .
$$

Thus, a straightforward calculation reveals that

$$
\begin{aligned}
\left\|\left(-\partial_{x}^{2}\right)^{s_{1} / 2}\left(-\partial_{y}^{2}\right)^{s_{2} / 2} \varphi_{c}\right\|_{L^{2}} & =c^{2 / k-3 / 2+2 s_{1}+s_{2}}\left\|\left(-\partial_{x}^{2}\right)^{s_{1} / 2}\left(-\partial_{y}^{2}\right)^{s_{2} / 2} \varphi_{1}\right\|_{L^{2}} \\
& =\left\|\left(-\partial_{x}^{2}\right)^{s_{1} / 2}\left(-\partial_{y}^{2}\right)^{s_{2} / 2} \varphi_{1}\right\|_{L^{2}}=\vartheta .
\end{aligned}
$$

Note that the constant $\vartheta$ does not depend on $c$.

Next, for any $c>0$ fixed, we consider

$$
u_{c}(x, y, t)=\varphi_{c}(x-c t, y) .
$$

Hence, at $t=0$, we have $u_{c}(0)=\varphi_{c}$. Moreover, for any $c_{1}, c_{2}>0$, we obtain

$$
\begin{array}{r}
\left\|\left(-\partial_{x}^{2}\right)^{s_{1} / 2}\left(-\partial_{y}^{2}\right)^{s_{2} / 2}\left(\varphi_{c_{1}}-\varphi_{c_{2}}\right)\right\|_{L^{2}}^{2}=\left\|\left(-\partial_{x}^{2}\right)^{s_{1} / 2}\left(-\partial_{y}^{2}\right)^{s_{2} / 2} \varphi_{c_{1}}\right\|_{L^{2}}^{2} \\
+\left\|\left(-\partial_{x}^{2}\right)^{s_{1} / 2}\left(-\partial_{y}^{2}\right)^{s_{2} / 2} \varphi_{c_{2}}\right\|_{L^{2}}^{2}-2\left\langle\varphi_{c_{1}}, \varphi_{c_{2}}\right\rangle_{s_{1}, s_{2}} .
\end{array}
$$

But, we find that

$$
\begin{aligned}
\left\langle\varphi_{c_{1}}, \varphi_{c_{2}}\right\rangle_{s_{1}, s_{2}} & =\int_{\mathbb{R}^{2}}\left(-\partial_{x}^{2}\right)^{s_{1} / 2}\left(-\partial_{y}^{2}\right)^{s_{2} / 2} \varphi_{c_{1}}(x, y) \overline{\left(-\partial_{x}^{2}\right)^{s_{1} / 2}\left(-\partial_{y}^{2}\right)^{s_{2} / 2} \varphi_{c_{2}}}(x, y) d x d y \\
& =\int_{\mathbb{R}^{2}}|\xi|^{2 s_{1}}|\eta|^{2 s_{2}} \widehat{\varphi_{c_{1}}}(\xi, \eta) \overline{\widehat{\varphi_{c_{2}}}}(\xi, \eta) d \xi d \eta \\
& =\left(c_{1} c_{2}\right)^{\frac{1}{k}-\frac{3}{2}} \int_{\mathbb{R}^{2}}|\xi|^{2 s_{1}}|\eta|^{2 s_{2}} \widehat{\varphi_{1}}\left(\frac{\xi}{c_{1}}, \frac{\eta}{\sqrt{c_{1}}}\right) \overline{\widehat{\varphi_{1}}}\left(\frac{\xi}{c_{2}}, \frac{\eta}{\sqrt{c_{2}}}\right) d \xi d \eta \\
& =\left(\frac{c_{2}}{c_{1}}\right)^{\frac{1}{k}-\frac{3}{2}} \int_{\mathbb{R}^{2}}|\xi|^{2 s_{1}}|\eta|^{2 s_{2}} \widehat{\varphi_{1}}(\xi, \eta) \widehat{\widehat{\varphi_{1}}}\left(\frac{c_{1}}{c_{2}} \xi, \sqrt{\frac{c_{1}}{c_{2}}} \eta\right) d \xi d \eta
\end{aligned}
$$

Therefore, as $\theta:=c_{1} / c_{2} \rightarrow 1$, we get

$$
\lim _{\theta \rightarrow 1}\left\langle\varphi_{c_{1}}, \varphi_{c_{2}}\right\rangle_{s_{1}, s_{2}}=\vartheta^{2} .
$$

As a consequence of (4.3)-(4.5), we then get

$$
\lim _{\theta \rightarrow 1}\left\|\left(-\partial_{x}^{2}\right)^{s_{1} / 2}\left(-\partial_{y}^{2}\right)^{s_{2} / 2}\left(\varphi_{c_{1}}-\varphi_{c_{2}}\right)\right\|_{L^{2}}=0 .
$$

On the other hand, for any $t>0$,

$$
\begin{gathered}
\left\|\left(-\partial_{x}^{2}\right)^{s_{1} / 2}\left(-\partial_{y}^{2}\right)^{s_{2} / 2}\left(u_{c_{1}}(t)-u_{c_{2}}(t)\right)\right\|_{L^{2}}^{2}=\left\|\left(-\partial_{x}^{2}\right)^{s_{1} / 2}\left(-\partial_{y}^{2}\right)^{s_{2} / 2} u_{c_{1}}(t)\right\|_{L^{2}}^{2} \\
+\left\|\left(-\partial_{x}^{2}\right)^{s_{1} / 2}\left(-\partial_{y}^{2}\right)^{s_{2} / 2} u_{c_{2}}(t)\right\|_{L^{2}}^{2}-2\left\langle u_{c_{1}}(t), u_{c_{2}}(t)\right\rangle_{s_{1}, s_{2}} .
\end{gathered}
$$

But, since

$$
\widehat{u_{c}(t)}(\xi, \eta)=c^{1 / k-3 / 2} e^{-i c \xi t} \widehat{\varphi}_{1}\left(\frac{\xi}{c}, \frac{\eta}{\sqrt{c}}\right)
$$


we deduce that

$$
\begin{aligned}
& \left\langle u_{c_{1}}(t), u_{c_{2}}(t)\right\rangle_{s_{1}, s_{2}} \\
& =\int_{\mathbb{R}^{2}}\left(-\partial_{x}^{2}\right)^{s_{1} / 2}\left(-\partial_{y}^{2}\right)^{s_{2} / 2} \varphi_{c_{1}}\left(x-c_{1} t, y\right) \overline{\left(-\partial_{x}^{2}\right)^{s_{1} / 2}\left(-\partial_{y}^{2}\right)^{s_{2} / 2} \varphi_{c_{2}}}\left(x-c_{2} t, y\right) d x d y \\
& =\left(c_{1} c_{2}\right)^{\frac{1}{k}-\frac{3}{2}} \int_{\mathbb{R}^{2}} e^{-i t \xi\left(c_{1}-c_{2}\right)}|\xi|^{2 s_{1}}|\eta|^{2 s_{2}} \widehat{\varphi_{1}}\left(\frac{\xi}{c_{1}}, \frac{\eta}{\sqrt{c_{1}}}\right) \overline{\widehat{\varphi_{1}}}\left(\frac{\xi}{c_{2}}, \frac{\eta}{\sqrt{c_{2}}}\right) d \xi d \eta \\
& =\left(\frac{c_{2}}{c_{1}}\right)^{\frac{1}{k}-\frac{3}{2}} \int_{\mathbb{R}^{2}} e^{-i t \xi c_{1}\left(c_{1}-c_{2}\right)}|\xi|^{2 s_{1}}|\eta|^{2 s_{2}} \widehat{\varphi_{1}}(\xi, \eta) \overline{\varphi_{1}}\left(\frac{c_{1}}{c_{2}} \xi, \sqrt{\frac{c_{1}}{c_{2}}} \eta\right) d \xi d \eta .
\end{aligned}
$$

Choosing $c_{1}=m+1$ and $c_{2}=m \in \mathbb{N}$ and letting $m \rightarrow \infty$, then by the RiemannLebesgue lemma, we obtain

$$
\lim _{m \rightarrow \infty}\left\langle u_{c_{1}}(t), u_{c_{2}}(t)\right\rangle_{s_{1}, s_{2}}=0 .
$$

Therefore, for any $t>0$,

$$
\lim _{\theta \rightarrow 1}\left\|\left(-\partial_{x}^{2}\right)^{s_{1} / 2}\left(-\partial_{y}^{2}\right)^{s_{2} / 2}\left(u_{c_{1}}(t)-u_{c_{2}}(t)\right)\right\|_{L^{2}}=\sqrt{2} \vartheta
$$

This completes the proof of the theorem.

\section{ACKNOWLEDGMENT}

This paper was written when the second author had a post-doctoral position at IMPA/Brazil. He gratefully acknowledges IMPA's hospitality.

\section{REFERENCES}

1. J. P. Albert, Concentration compactness and stability of solitary-wave solutions to nonlocal equations, Contemp. Math. 221, Amer. Math. Soc., Providence, RI, 1999, 1-29. MR1647189 (99m:35199)

2. J. Angulo, Nonlinear dispersive equations. Existence and stability of solitary and periodic travelling wave solutions, Math. Surveys Monogr. 156, Amer. Math. Soc., Providence, RI, 2009. MR2567568

3. H. Biagioni and F. Linares, Ill-posedness for the derivative Schrödinger and generalized Benajamin-Ono equations, Trans. Amer. Math. Soc 353 (2001), 3649-3659. MR.1837253 (2002e:35215)

4. B. Birnir, G. Ponce, and N. Svanstedt, The local ill-posedness of the modified KdV equation, Ann. Inst. H. Poincaré Anal. Non Linéaire 13 (1996), 529-535. MR1404320 (97e:35152)

5. B. Birnir, C. E. Kenig, G. Ponce, N. Svanstedt, and L. Vega, On the ill-posedness of the IVP for the generalized Korteweg-de Vries and nonlinear Schrödinger equations, J. London Math. Soc. 53 (1996), 551-559. MR1396718 (97d:35233)

6. J. Bourgain, Periodic Korteweg-de Vries equation with measures as initial data, Selecta Math. 3 (1997), 115-159. MR 1466164 (2000i:35173)

7. N. Burq and F. Planchon, On well-posedness for the Benjamin-Ono equation, Math. Ann. 340 (2008), 497-542. MR2357995 (2009a:35205)

8. A. Esfahani and A. Pastor, Instability of solitary wave solutions for the generalized BO-ZK equation, J. Differential Equations 247 (2009), 3181-3201. MR2571573

9. A. Esfahani, A. Pastor, and J. Bona, Stability and decay properties of solitary wave solutions for the generalized $B O-Z K$ equation, preprint.

10. A. Esfahani and A. Pastor, Stability of solitary waves for the generalized Shrira equation, in preparation.

11. D. G. Gaidashev and S. K. Zhdanov, On the transverse instability of the two-dimensional Benjamin-Ono solitons, Phys. Fluid 16 (2004), 1915-1921. MR.2060293 (2004m:76070)

12. M. Hadac, Well-posedness for the Kadomtsev-Petviashvili II equation and generalisations, Trans. Amer. Math. Soc. 360 (2008), 6555-6572. MR2434299 (2009g:35265) 
13. A. D. Ionescu and C. E. Kenig, Global well-posedness of the Benjamin-Ono equation in lowregularity spaces, J. Amer. Math. Soc. 20 (2007), 753-798. MR2291918 (2008f:35350)

14. A. D. Ionescu and C. E. Kenig, Local and global well-posedness of periodic KP-I equations, Ann. of Math. Stud. 163, Princeton University Press, Princeton, NJ, 2007, 181-211. MR 2333212 (2009g:35269)

15. M. C. Jorge, G. Cruz-Pacheco, L. Mier-y-Teran-Romero, and N. F. Smyth, Evolution of twodimensional lump nanosolitons for the Zakharov-Kuznetsov and electromigration equations, Chaos 15 (2005), 037104-1 - 037104-13. MR2184880(2006f:35244)

16. C. E. Kenig, On the local and global well-posedness theory for the KP-I equation, Ann. Inst. H. Poincaré Anal. Non Linéaire 21 (2004), 827-838. MR2097033 (2005j:35196)

17. J. C. Latorre, A. A. Minzoni, N. F. Smyth, and C. A. Vargas, Evolution of Benjamin-Ono solitons in the presence of weak Zakharov-Kutznetsov lateral dispersion, Chaos 16 (2006), 043103-1-043103-10. MR2289280 (2007i:35200)

18. F. Linares and J.-C. Saut, The Cauchy problem for the 3D Zakharov-Kuznetsov equation, Discrete Contin. Dyn. Syst. 24 (2009), 547-565. MR2486590 (2010e:35242)

19. A. Milanés, Some results about a bidimensional version of the generalized $B O$, Commun. Pure Appl. Anal. 2 (2003), 233-250. MR.1975062 (2004c:35363)

20. A. Milanés, On some bidimensional versions of the generalized Benjamin-Ono equation, Ph.D. Thesis, IMPA, 2001.

21. L. Molinet and F. Ribaud, Well-posedness results for the generalized Benjamin-Ono equation with small initial data, J. Math. Pures Appl. (9) 83 (2004), 277-311. MR2038121 (2005a:35252)

22. L. Molinet, F. Ribaud, and A. Youssfi, Ill-posedness issues for a class of parabolic equations, Proc. Roy. Soc. Edinburgh Sect. A 132 (2002), 1407-1416. MR.1950814 (2003m:35240)

23. L. Molinet, J.-C. Saut, and N. Tzvetkov, Ill-posedness issues for the Benjamin-Ono and related equations, SIAM J. Math. Anal. 33 (2001), 982-988. MR.1885293 (2002k:35281)

24. L. Molinet, J.-C. Saut, and N. Tzvetkov, Well-posedness and ill-posedness results for the Kadomtsev-Petviashvili-I equation, Duke Math. J. 115 (2002), 353-384. MR 1944575 (2003h:35240)

25. D. E. Pelinovsky and V. I. Shrira, Collapse transformation for self-focusing solitary waves in boundary-layer type shear flows, Physics Letters A 308 (1995), 195-202.

26. V. I. Shrira, On surface waves in the upper quasi-uniform ocean layer, Dokl. Akad. Nauk SSSR 308 (1989), 732-736.

27. N. Tzvetkov, Remark on the local ill-posedness for KdV equation, C. R. Acad. Sci. Paris Sér. I Math. 329 (1999), 1043-1047. MR.1735881 (2001f:35359)

28. T. Tao, Global well-posedness of the Benjamin-Ono equation in $H^{1}(\mathbb{R})$, J. Hyperbolic Differ. Equ. 1 (2004), 27-49. MR2052470 (2005f:35273)

Department of Mathematics, IME-USP, Rua do Matão 1010, Cidade Universitária, 05508-090, SÃo PAUlo, SP, Brazil

E-mail address: amin@impa.br

Current address: School of Mathematics and Computer Science, Damghan University of Basic

Sciences, Damghan, 36716-41167, Iran

E-mail address: esfahani@dubs.ac.ir

imeCC-UniCAmP, Cidade Universitária, Rua Sérgio Buarque de Holanda, 651, 13083-859, CAMPINAS, SP, BRAZIL

E-mail address: apastor@ime.unicamp.br 